\title{
Spontaneous Fracture of the Tibial Sesamoid: A case report
}

\author{
by Al Kline, $\mathrm{DPM}^{1} \square$
}

\section{The Foot \& Ankle Journal 1 (9): 3}

A tibial sesamoid fracture is described. A female, while walking in an airport, experiences discomfort and pain under the first metatarsal head while wearing a high-heel shoe. Clinical investigation reveals a spontaneous fracture of the tibial sesamoid. The patient also has associated hallux valgus. Conservative treatment and surgical options are presented and discussed. The patient, after failed conservative treatment, had the sesamoid removed and a simple bunionectomy performed. It appears that a centrally placed tibial sesamoid with associated hallux valgus can cause a spontaneous fracture of the tibial sesamoid under the right circumstances.

Key words: Tibial sesamoid fracture, HAV deformity, bunion

Accepted: August 2008

Published: September 2008

This is an Open Access article distributed under the terms of the Creative Commons Attribution License. It permits unrestricted use, distribution, and reproduction in any medium, provided the original work is properly cited. @The Foot \& Ankle Journal (www.faoj.org)

$\mathbf{T}$ ibial sesamoid fracture is common in athletes and active individuals. Usually, the incident is preceded by stress injury or acute trauma from stressed dorsiflexion or hyperextension of the hallux. The first ray will transmit about $60 \%$ of a person's weight-bearing stress through the first metatarsophalangeal joint during the gait cycle. In acute instances, these stresses can be many times greater. Interestingly, there have been infrequent reports of tibial sesamoid fracture from non-acute injury such as walking. In non-acute injuries, many patients will present with a sharp initial pain followed by a dull ache or pain under the sesamoids. The differential diagnosis may include sesamoiditis, chondromalacia, first metatarsal joint capsulitis, flexor hallucis tendonitis and arthritis.

Address correspondence to: Al Kline, DPM

3130 South Alameda, Corpus Christi, Texas 78404.

${ }^{1}$ Adjunct Clinical Faculty, Barry University School of Podiatric Medicine. Private practice, Chief of Podiatry, Doctors Regional Medical Center. Corpus Christi, Texas, 78411.
MRI and radiographic evaluation are the most common modalities used to differentiate injury and fracture from more subtle injuries such as sesamoiditis or capsulitis.

A clinical entity such as a bipartite sesamoid may confuse the clinician in the proper diagnosis. This condition was first described as early as 1904 by Stieda and confirmed by Dwight. ${ }^{1}$ Bipartite sesamoids are a common finding on foot radiographs. They often look like tibial sesamoid fractures, however, close inspection of the radiograph usually reveals a well-defined, smoother partition between the two fragments. A fractured sesamoid will likely have a rough, irregular partition. Bipartite sesamoids are a normal variant when two ossification centers fail to fuse at maturity. This condition is present in about $10 \%$ of the population, and in that population, $25 \%$ are bilateral. ${ }^{2}$ In cases of acute injury, diastasis of the bipartite sesamoid can lead to acute pain under the first metatarsal head. This has been reported in hyperextension or "turf-toe" injuries in football players. ${ }^{3}$ 
It is the author's opinion that bipartite sesamoids can also lead to sesamoid pain. Bone scintigraphy can also differentiate sesamoid fracture from congenital partition. ${ }^{4}$

In non-acute injuries of the tibial sesamoid, conservative treatments are designed to de-weight the sesamoid and allow for healing. Conservative methods of treatment can include wearing a surgical shoe to prevent extension of the toe or even casting and non-weightbearing. Once the correct diagnosis is made, appropriate treatment can be initiated. Conservative care for sesamoiditis can also include wearing an accommodative insert with first metatarsal padding. The pad can be placed just behind the sesamoid in order to decrease stress through the sesamoid apparatus. Other conservative treatments include 1) NSAIDS, 2) avoiding high heels, 3) off-loading shoes and 4) steroid injection. These injections may be used along with joint taping to prevent excessive extension of the first metatarsophalangeal joint. Care must be taken in the injection of steroids to this region, especially if fracture is suspected. Injection of steroids can sometimes lead to avascular necrosis of a fractured tibial sesamoid.

In acute fracture, non-weightbearing in a cast is recommended for 6 to 8 weeks. ${ }^{5,7}$ It appears, from experience and previous study, that the nonunion rate of sesamoid fractures is high. This may be related to the vascular supply of the sesamoids and the forces placed on the bone fragments once fractured. It is theorized that the anatomical pull of soft tissue, once the sesamoid fractures, causes a distraction of the fracture line. Anatomically, the tibial sesamoid has anatomical attachments to the abductor hallucis muscle, plantar fascia, flexor hallucis brevis tendon, intersesamoidal ligament, medial metatarsal and phalangeal sesamoid ligaments. ${ }^{4}$

Once the tibial sesamoid fractures, these attachments tend to distract the fracture fragments. The vascularity of the tibial sesamoid is supplied by three sources: 1) the deep and superficial branches of the medial plantar artery,
2) branches from the lateral plantar artery and perforating branches of the dorsalis pedis artery, and 3) pole arteries through the length of the sesamoid. ${ }^{4,6}$ One explanation for the high incidence of non-union is that the primary blood supply of all three sources originates from the proximal pole of the sesamoid through the tendon of the flexor hallucis brevis. ${ }^{4}$ Since most fractures of the tibial sesamoid are transverse, once the fracture occurs, the distraction causes disruption of this vascular communication leading to non-union. Bone stimulators may also be effective in the conservative treatment of tibial sesamoid fractures. They are certainly indicated after immobilization and non-unions are suspected.

\section{Anatomy and Sesamoid Position in its relation to Hallux Valgus}

The sesamoid position may also play an important role in stresses that are directed through the metatarsophalangeal joint. Anatomically, both the tibial and fibular sesamoids act in unison during the propulsive phase of gait. The articular surfaces of both the tibial and fibular sesamoids glide along the sesamoidal groove and are separated by the central crista in its anatomical alignment. The crista is a central plantar ridge along the metatarsal head that separates the sesamoids. The glistening surfaces of the sesamoids are facet- shaped within the plantar groove of the metatarsal head. In hallux valgus, the sesamoid position is described in distinctive positions. These positions are described as positions 1-7 in the radiographic evaluation of hallux valgus. (Fig. 1.)

It is commonly understood that these positions are dictated by the movement of the metatarsal head as the entire metatarsal subluxes medially in the classic hallux abductovalgus deformity.

It is theorized that position four is the most stressful position for the tibial sesamoid and could cause tibial sesamoid fracture under the right circumstances. 


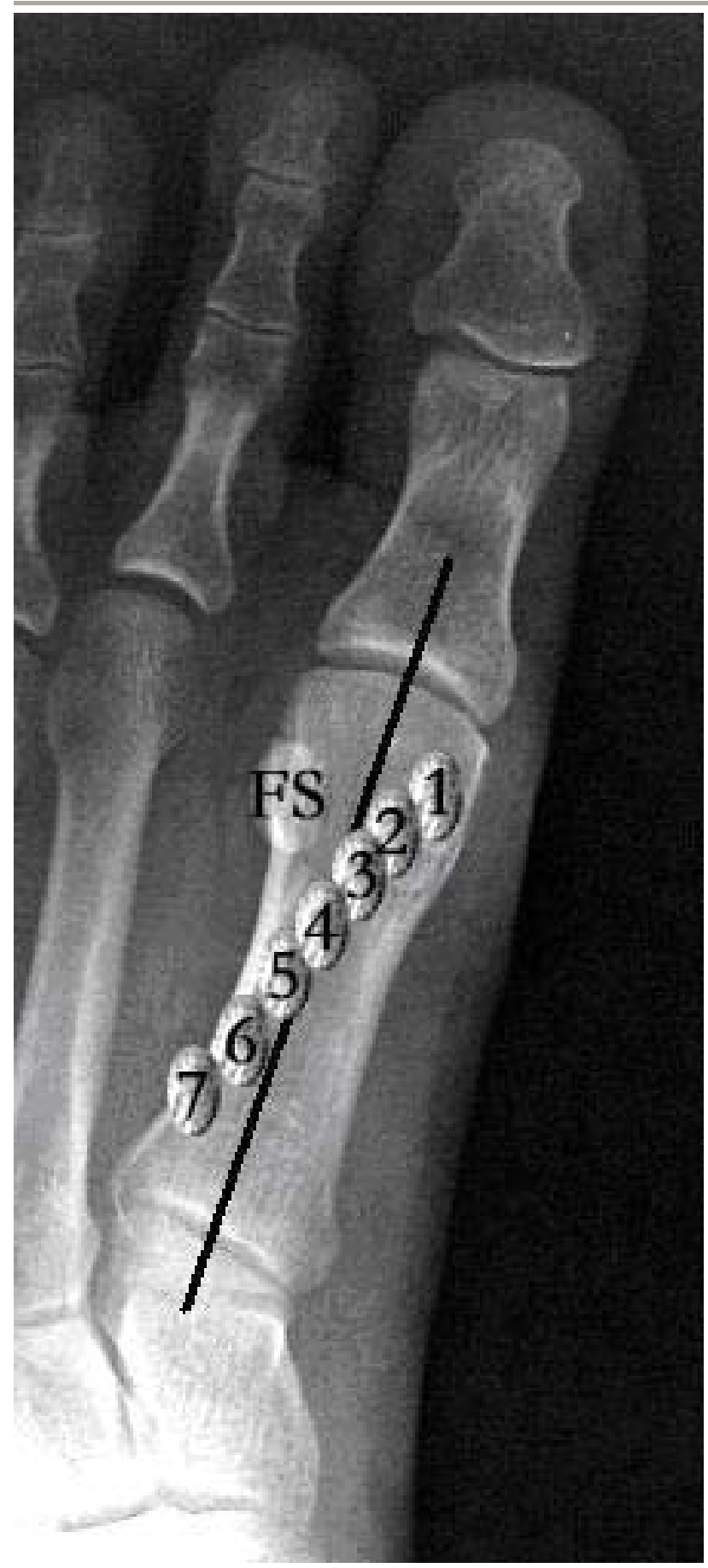

Figure 1 Tibial sesamoid position is radiographically described in seven positions. Position four is described as being directly under the crista of the first metatarsal head, likely increases stress forces through the tibital sesamoid. The fibular sesamoid (FS) will rotate even further into the first interspace between the first and second metatarsal as the hallux valgus worsens.

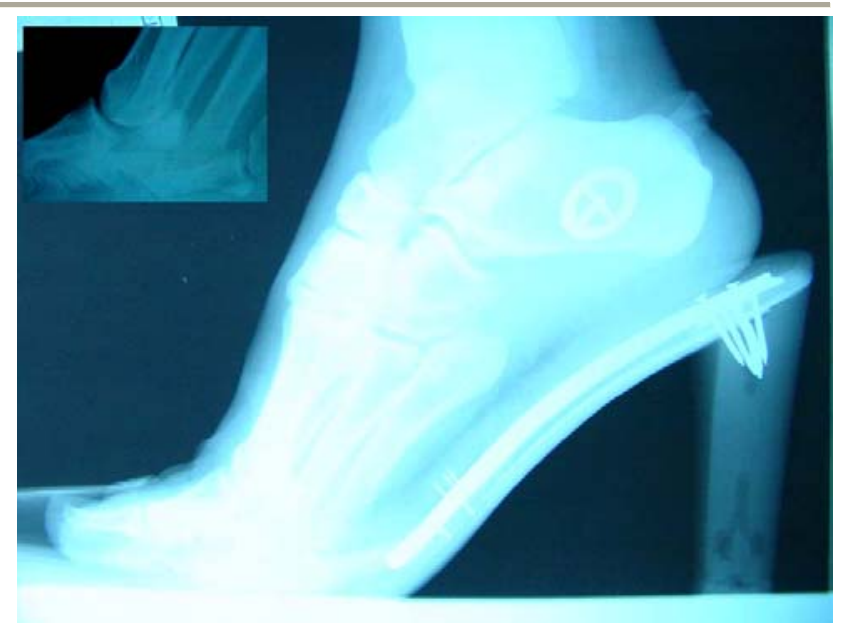

Figure 2 Radiographs demonstrate the increased hyperextension of the first metatarsophalangeal joint in a high-heel shoe. It is theorized that excessive hyperextension of the joint places increased pressure through the sesamoid apparatus.

This may be even more plausible in a high-heeled shoe that causes increased stress along the plantar metatarsophalangeal joint. (Fig. 2)

\section{Case Report}

In February 2006, a 33 year old active female began to experience episodes of pain under the tibial sesamoid. She reported walking in the airport and experiencing a pain under the first metatarsophalangeal joint while in a high heel. She initially presented to another podiatrist, who diagnosed her with 'fractured tibial sesamoid'. He instituted conservative treatment including padding and a local steroid injection. She did well for several months, but continued to have episodes of increasing pain.

She presented to our office in September 2006. Clinical evaluation revealed bunion pain and pain directly under the tibial sesamoid. She was ambulating and wearing shoes, but had episodes of pain and signs of non-healing of the tibial sesamoid fracture. Radiographs confirmed a tibial sesamoid fracture with a mild bunion deformity. (Fig. 3) 


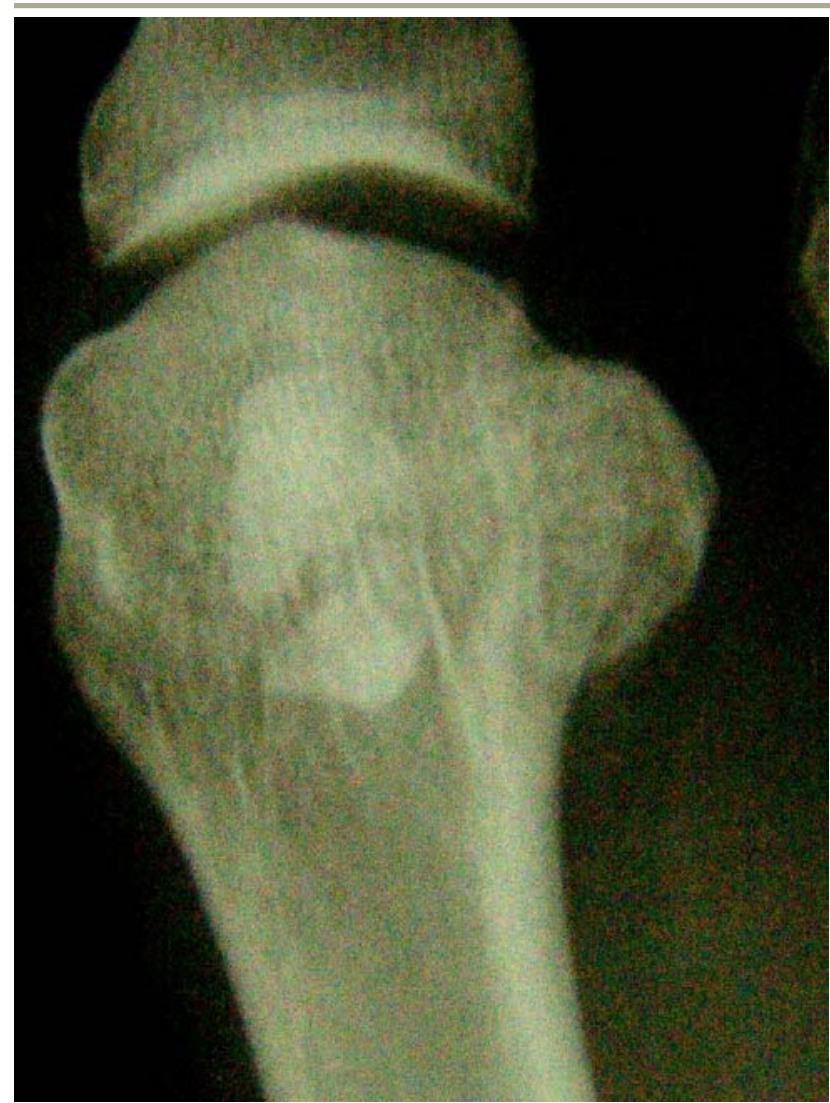

Figure 3 Radiographs confirm tibial sesamoid fracture. The fracture edges are irregular and sharp, consistent with fracture instead of bi-partite sesamoid. The tibial sesamoid is positioned directly under the crista in position sesamoid four.

We placed her on crutches with off-loading for 6 weeks and progression to a Darco ${ }^{\text {TM }}$ walking shoe, then athletic sneakers with proper accommodative metatarsal padding. Despite these attempts, she continued to have pain and we decided to schedule her for simple bunionectomy and tibial sesamoid excision.

\section{Surgical Technique}

The patient was brought to the operating room and underwent simple bunionectomy and tibial sesamoid excision. A medial incision is made along the border of the first metatarsophalangeal joint. Care must be taken to identify the medial cutaneous nerve or 'bunion' nerve.

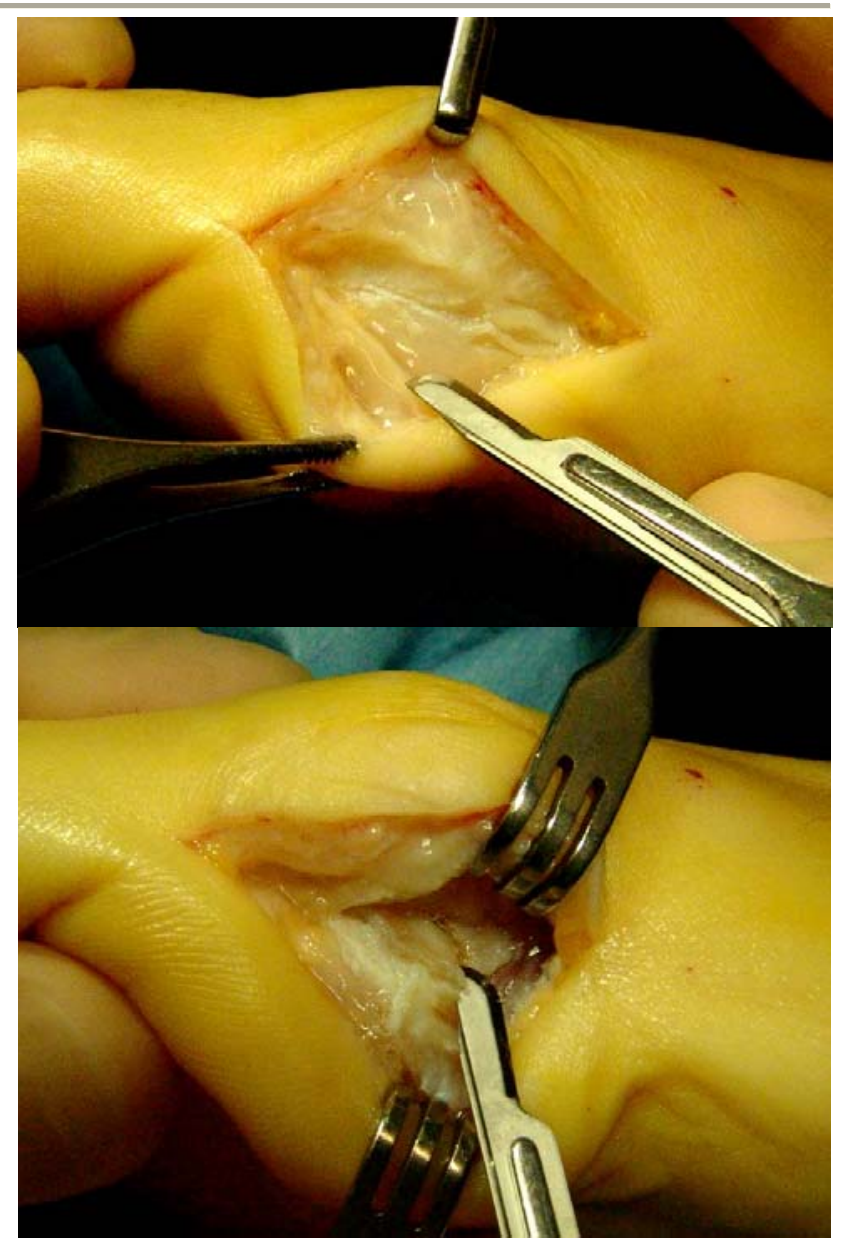

Figures 4ab (a) A medially placed incision along the sesamoid is fashioned. Care is taken to identify the medial cutaneous nerve. (identified at the tip of the blade). (b) Once the capsule is incised, the fractured tibial sesamoid is identified.

The medial cutaneous nerve has variable branches and may run a course more medial and inferior to the dorsomedial branch. Once the nerve is identified, a medical capsular incision is made and the fracture is identified. (Figs. 4ab) Care must also be taken when excising the tibial sesamoid. The sesamoid is encased along the capsular tissue of the first metatarsophalangeal joint. It has attachments to the abductor hallucis muscle, plantar fascia, flexor hallucis brevis tendon, intersesamoidal ligament, medial metatarsal and phalangeal sesamoid ligaments. The flexor hallucis longus tendon also glides between the two sesamoids. 


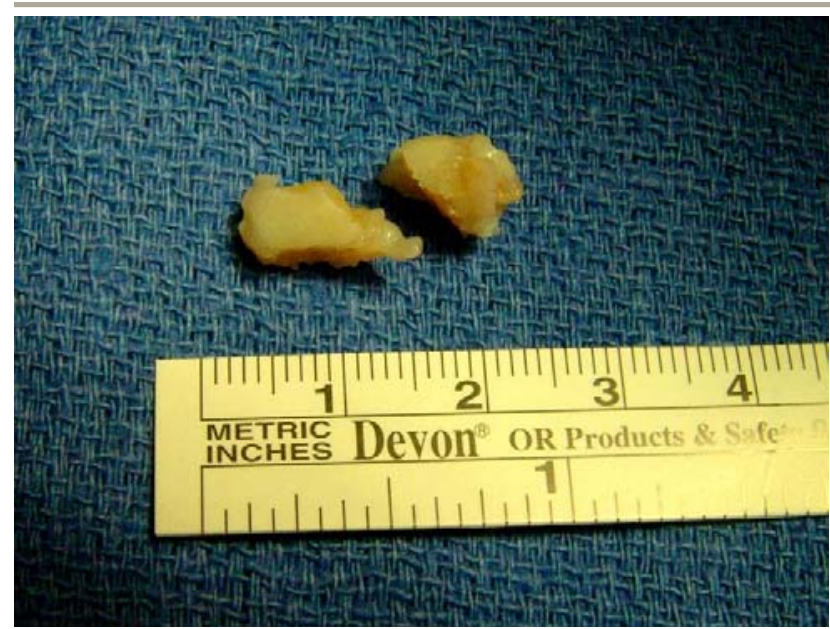

Figure 5 After the tibial sesamoid fracture is excised, it appears as if the fracture recently occurred. There are no signs of attempted bone healing or calcification. This is testament to the distraction forces that keep the sesamoid fracture apart.

Care must be taken not to cut the tendon when excising the sesamoid. A number 15 blade is used to enucleate the sesamoid from these fibrous, soft tissue attachments. Once the sesamoid is removed, suture is used to approximate the ligamentous and capsular attachments. The sesamoid should be 'cleanly' excised with little tissue attachment. (Fig. 5) The postoperative radiographs reveal a post-excisional tibial sesamoidectomy with simple bunionectomy. (Fig. 6).

\section{Discussion}

Surgical excision of the entire tibial sesamoid appears to be a viable alternative of treatment to non-healing fractures of the tibial sesamoid. One of the earliest reports of tibial sesamoidectomy with relief of symptoms was described by Müller in 1911. ${ }^{1}$ Recently, Jones et al., reported the results of tibial sesamoid excision following tibial sesamoid fracture in a softball player. After 1 year, the patient was "free of pain in all shoe types". There is only one report to date of attempting internal fixation to repair the tibial sesamoid fracture.

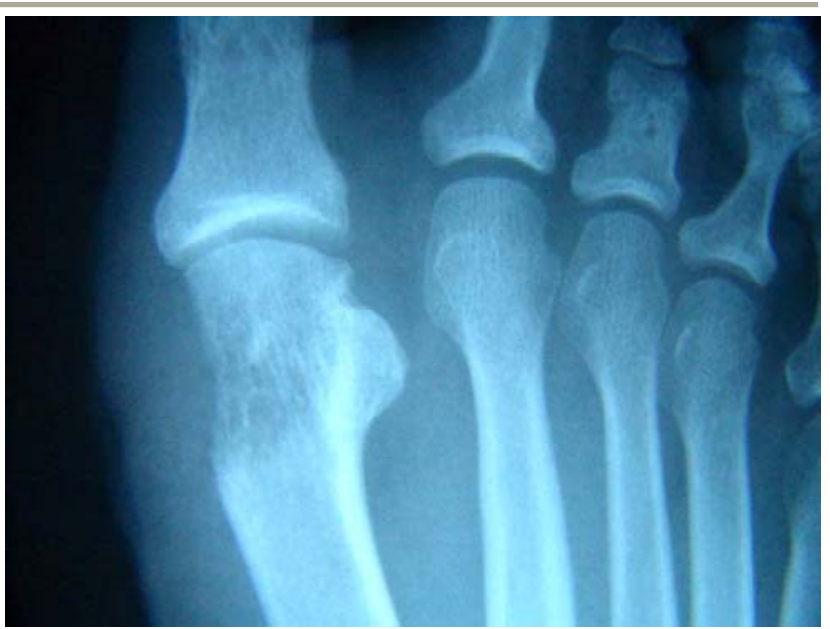

Figure 6 Post-operative radiographs reveal removal of the tibial sesamoid and a simple bunionectomy performed.

In 2001, Riley and Selner reported using monofilament wire to internally fixate a tibial sesamoid fracture with excellent results in a 17 year old female. 7 This approach may be more desirable in the acute fracture of young, active patients. In our case, surgical excision was more desirable since the tibial sesamoid was directly placed under the crista. Reported complications of removing the tibial sesamoid include hallux valgus, transfer pain, tendonitis, arthritis and hallux extensus. ${ }^{5,7}$

In this particular case, the association of hallux valgus and the placement of the tibial sesasmoid in position four appear to increase the likelihood of fracture when wearing a high-heeled shoe. The association of hallux valgus as a risk for tibial sesamoid fracture was first described in 1929, although most cases of fractures are related to direct trauma and a sudden increase in weightbearing force, Hobart reported "an association with hallux valgus is often found". Anatomically, the central rise of the crista may explain the increased force through a sesamoid. Stressed dorsiflexion in a high heeled shoe after extensive walking could directly lead to spontaneous fracture. In this case, our surgical goals were straight forward: simple bumpectomy with sesamoid removal. 
To date, the patient has returned to activity with accommodative sesamoid padding and without pain or discomfort.

\section{Conclusion}

The tibial sesamoid fracture is now demonstrated to be a common fracture of the foot. The diagnosis is usually made by clinical presentation, using radiographs and MRI for confirmation. Bone scans can also be used. The stresses placed through the metatarsal head during gait can be complicated. This report suggests that an abnormally placed sesamoid due to associated hallux valgus or bunion may lead to increased stress of the sesamoid and can lead to fracture. This is especially true in patients who attempt to walk for extended periods in a high-heel shoe. It is suggested that patients with hallux valgus wear a simple accommodative device, such as a dress orthotic, in an attempt to off-load the stress through the sesamoids. This case also demonstrates that if the sesamoid does fracture, total tibial sesamoidectomy is a viable procedure in cases of non-union or delayed healing.

\section{References}

1. Müller, G.P. Fracture of the Sesamoid Bones. Ann Surg, Read before the Philadelphia Academy of Surgery, October 2, 1911. [Online-PDF

2. Neerajana, DODA, Wilfred, C.G. PEH Woman with possible right toe fracture: Radiology Series Asia Pacific Journal of Family Medicine, Volume 5, Issue 3, 2006. Online: $[\underline{\mathrm{PDF}}]$.

3. Rodeo, SA, et al Diastasis of bipartite sesamoids of the first metatsophalangeal joint. Foot Ankle, 14(8): 425-34. Oct. 1993.

4. Swierzewski, J. Acute and Chronic Injuries to the Sesamoids: Etiology and Treatment CPMA, Vol 10, No. 4, Fall 2001. [Online]

5. Jones, J.L., Losito, J.M. Tibial Sesamoid Fracture in a Softball Player JAPMA Vol. 97, No. 1 Jan/Feb 2007.

6. Banks, A.S., Downey, M.S., et al McGlamry's Comprehensive Textbook of Foot and Ankle Surgery, 3rd ed. Lipcott and Williams, 2001.

7. Riley, J., Selner, M. Internal Fixation of a Displaced Tibial Sesamoid Fracture JAPMA, Vol. 91, No. 10, 2001.

8. Hobart, M. Fracture of Sesamoid Bones of the Foot: With Report of a Case JBJS (Am) 11:298-302, 1929. 